\title{
La Estructura Agroecológica Principal (EAP): novedosa herramienta para planeación del uso de la tierra en agroecosistemas
}

\section{The Principal Agroecological Structure (PAS): a new tool for planning agroecosystems}
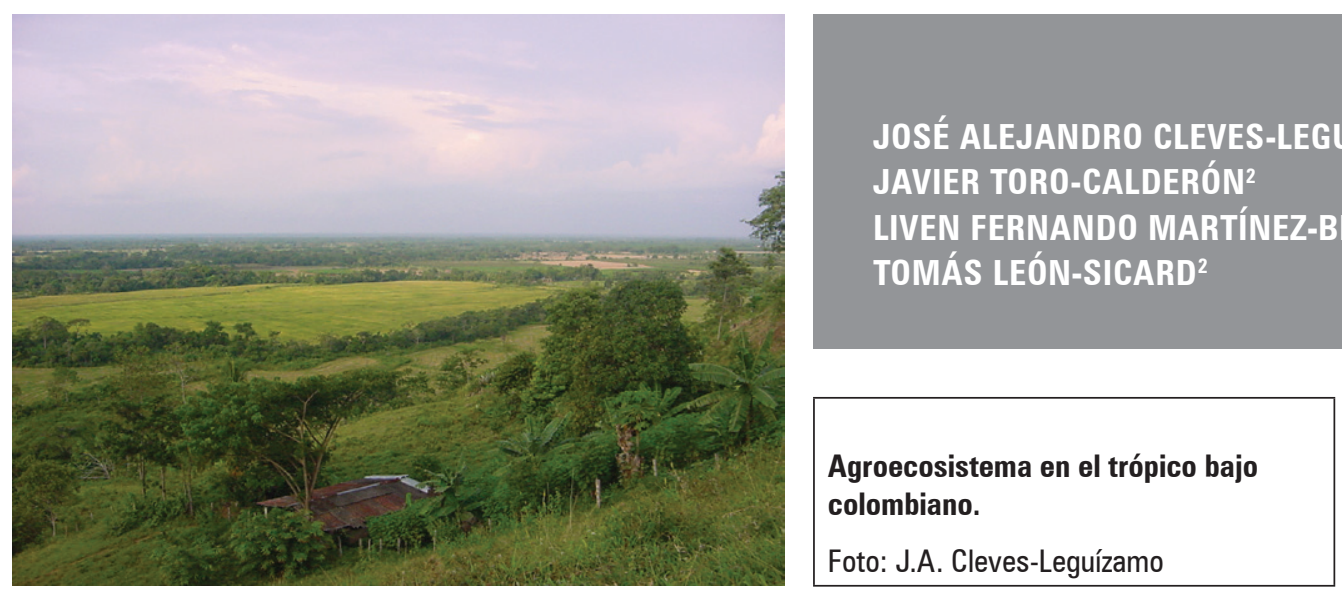

\section{RESUMEN}

Este documento presenta a partir del pensamiento ambiental (relaciones ecosistema-cultura): el concepto de la Estructura Agroecológica Principal (EAP), de los agroecosistemas mayores considerada como una estructura disipativa de orden cultural y se discute sus posibles aplicaciones (resiliencia, producción, diversidad) al interior como al exterior de las fincas. La EAP puede ser útil en la planificación del uso de la tierra, teniendo en cuenta el concepto de la EAP potencial (que permite cuantificar el manejo de los corredores internos y externos, incluyendo la vegetación natural). Al mismo tiempo, puede ser utilizada en el contexto del ordenamiento territorial, porque visibiliza una serie de relaciones culturales (económicas, sociales, simbólicas y tecnológicas) ocultas desde el análisis parcial de la ecología del paisaje.

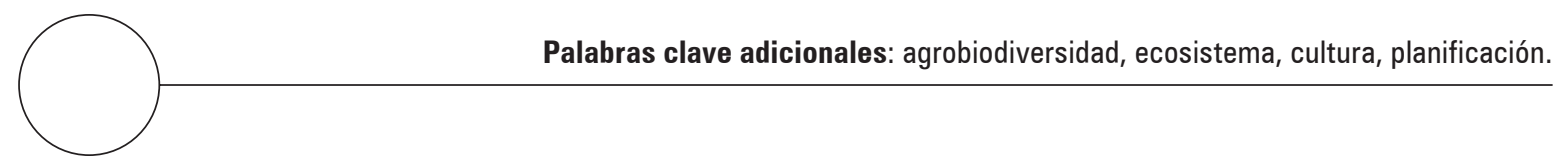

1 Facultad Seccional Duitama, Universidad Pedagógica y Tecnológica de Colombia (UPTC), Duitama (Colombia). ORCID Cleves-Leguízamo, J.A.: 0000-0001-9717-9753

2 Instituto de Estudios Ambientales (IDEA), Universidad Nacional de Colombia, Bogotá (Colombia). ORCID ToroCalderón, J.: 0000-0001-6675-5148; ORCID Martínez-Bernal, L.F.: 0000-0002-2117-3715; ORCID León-Sicard, T.: 0000-0001-6771-9253

3 Autor para correspondencia. jose.cleves@uptc.edu.co 


\section{ABSTRACT}

This document presents, from the environmental prespective (ecosystem-culture relationships), the concept of the Principal Agroecological Structure (PAS) of agroecosystems, considered a dissipative structure. It discusses possible applications (resilience, production, and diversity) both inside and outside farms. The PAS can be useful in land use planning on farms, based on the concept of potential PAS, which allows for the quantification of the management of internal and external corridors, including natural vegetation. At the same time, it can be useful in the context of landscape management because it shows a series of cultural relationships (economic, social, symbolic and technological), hidden from the partial analysis of landscape ecology.

Additional key words: agrobiodiversity, ecosystem, culture, planning.

Fecha de recepción: 03-04-2017 Aprobado para publicación: 30-09-2017

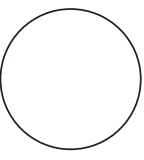

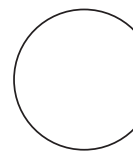

El pensamiento ambiental en esencia plantea y reconoce que la humanidad construyó un sistema de adaptación al entorno ecosistémico, diferente al de los demás seres vivos y basado fundamentalmente en la cultura. Donde se entiende la cultura como la herencia no biológica de la humanidad o, como todos aquellos procesos teóricos y prácticos que se expresan en las estructuras simbólicas, la organización social y las plataformas tecnológicas (Ángel, 1993; 1995; 1996). En el nivel cultural los sistemas de producción de alimentos se entienden como conjuntos de actividades que los grupos humanos organizan, dirigen y realizan de acuerdo con sus objetivos y recursos, influyendo en y, siendo influidos por el entorno ecosistémico y biofísico donde se localizan (Duarte, 1990). Aspecto que denota la relevancia y complejidad de la interacción ecosistema-cultura en el campo agrario (Ángel, 1996).

Por otra parte, es ampliamente aceptado que la agricultura es la resultante de la modificación cultural de los ecosistemas; cuando esas transformaciones aplican para la satisfacción de las necesidades básicas de alimento, fibras y otros materiales y se amplifican en función de los condicionantes de la coevolución ecosistema-cultura (León-Sicard, 2007; Fonseca y Cleves-Leguízamo 2015). El estudio de estas complejas relaciones es el objeto de la agroecología (León-Sicard, 2014), ciencia que se enfoca en el análisis ambiental de los agroecosistemas (Altieri y Nicholls, 2009; Altieri, 2009), reconociendo en ellos múltiples interacciones de tipo biofísico, social, económico, político y tecnológico (Altieri et al., 1999).
La agroecología estudia y propone respuestas a problemas complejos, a través de prácticas agrarias basadas en los conocimientos tradicionales o ancestrales, la promoción de la diversidad biológica y cultural, la autonomía de los productores y la conservación y manejo adecuado de los recursos naturales. Proponiendo un enfoque de carácter interdisciplinario en la investigación científica y estimulando con éxito las regulaciones biológicas, aún en agriculturas de pequeña escala (León-Sicard, 2010a; León-Sicard, 2010b; Méndez y Gliessman, 2002; Martínez, 2002; Toledo, 1990).

Dentro de estas prácticas o estrategias que surgen desde la agroecología, León-Sicard (2010a) propone estudiar lo que denominó: la Estructura Agroecológica Principal de los agroecosistemas mayores (EAP) ${ }^{1}$. Concepto que se refiere a los arreglos de los conectores internos y externos de las fincas y que podría estar relacionado con las probabilidades de resiliencia o adaptación de los sistemas agrarios a los disturbios de diferente naturaleza. Pudiéndose asociar teóricamente con una Estructura Disipativa (ED), con posibles efectos benéficos sobre el establecimiento y desarrollo de cultivos en diferentes escalas, desde la agricultura de subsistencia en áreas pequeñas hasta, los procesos productivos intensivos de carácter agroindustrial.

\footnotetext{
El autor distingue ecosistemas menores (cultivos, praderas, sitios forestales) y agroecosistemas mayores (fincas, haciendas, cooperativas o cualquier unidad productiva agropecuaria regida por derechos de propiedad o de tenencia).
} 
Igualmente, la EAP, podría permitir el diseño e implementación de opciones de adaptación y mitigación al clima cambiante, como aporte a la gestión del riesgo.

\section{CONCEPTOS PREVIOS}

\section{El agroecosistema}

El concepto de "sistema" es, en sí mismo de carácter multivariado, puesto que incluye la idea de múltiples componentes o subsistemas que interactúan en el tiempo y en el espacio, así como por su relación con suprasistemas (Escobar y Berdegué, 1990). El agroecosistema, es un ecosistema deliberadamente modificado por los seres humanos con el fin de obtener bienes y servicios, con un objetivo o fin económico y por lo tanto, es el lugar donde se presentan relaciones dinámicas entre la cultura y su entorno físico-biológico (Gliessman, 1990; Dalgaard et al., 2003).

Los agroecosistemas son considerados por varios autores, como unidades de análisis en donde confluyen tanto distintos tipos de procesos ecosistémicos (flujos energéticos, ciclos materiales) como de relaciones socioeconómicas (Altieri y Nicholls, 2009; Altieri, 2009).

Por otra parte, León-Sicard (2010a) definió el agroecosistema como: "el conjunto de relaciones e interacciones entre suelos, climas, plantas cultivadas, organismos de distintos niveles tróficos, plantas adventicias y grupos humanos en determinados espacios físicos y geográficos, cuando son enfocadas desde el punto de vista de sus flujos energéticos y de información, de sus ciclos materiales y de sus relaciones simbólicas, sociales, económicas y políticas, que se expresan en distintas formas tecnológicas de manejo dentro de contextos culturales específicos". Así mismo, las interacciones entre los componentes se presentan dentro de límites difusos que pueden ir más allá del predio o finca (Altieri et al., 1999).

\section{La Estructura Agroecológica Principal (EAP) de la finca 0 agroecosistema mayor}

El origen de la EAP se deriva de los trabajos de Van der Hammen (1998) y de Van der Hammen y Andrade (2003), quienes propusieron la idea de la Estructura Ecológica de Soporte de la Nación (EES), para facilitar la comprensión a escala del país del estado actual de sus coberturas vegetales. En esta EES los autores reconocieron dos componentes: la Estructura Ecológica Principal del Paisaje (EEP) y la Infraestructura Ecológica (IE). Y en ésta última incluyeron los remanentes de vegetación en los agroecosistemas, aunque no profundizaron en su significado y aplicaciones.

Con estos conceptos, los autores le dieron sentido al estudio de la ecología del paisaje y suministraron un trasfondo para articular, en la teoría y la práctica, el manejo de relictos y parches de vegetación natural, incluyendo corredores biológicos y masas boscosas. Además, en el concepto de Infraestructura Ecológica, introdujeron aquella vegetación existente en los agroecosistemas, pero no avanzaron en la formulación de esta idea.

Para dar cabida a esa relación inconclusa propuesta por los profesores van der Hammen y Andrade y para proporcionar un marco de referencia a la agroecología en función de una propiedad natural de los agroecosistemas: su propia estructura; nace el concepto de la Estructura Agroecológica Principal de los agroecosistemas,

León-Sicard (2010a) definió entonces la EAP como "la configuración o arreglo espacial interno de la finca y la conectividad entre sus distintos sectores, parches y corredores de vegetación o sistemas productivos, que permite el movimiento y el intercambio de distintas especies animales y vegetales, les ofrece refugio, hábitat y alimento, provee regulaciones microclimáticas e incide en la producción, conservación de recursos naturales y en otros aspectos ecosistémicos y culturales de los agroecosistemas mayores".

El agroecosistema mayor (finca) posee un contenido ecosistémico que se expresa en los agroecosistemas menores (lotes, sitios de cultivo, áreas forestales, agroforestales o silvopastoriles). La forma en que estos se relacionan con bosques u otros tipos de coberturas vegetales arbóreas, herbáceas o de matorral dentro del agroecosistema mayor, le imprime características determinadas a la EAP de la finca. Este concepto, que pretende describir las relaciones estructurales y funcionales de los agroecosistemas mayores, a la luz de los factores culturales que las determinan, puede ser utilizado para distintas aplicaciones (relaciones con producción, sanidad vegetal, autonomía alimentaria, resiliencia) incluyendo futuros usos como criterio taxonómico de agroecosistemas (León-Sicard, 2014). 
En los procesos de diseñar agroecosistemas resilientes a distintos tipos de disturbio, se requiere la comprensión de la complejidad inherente a los agroecosistemas (Altieri y Koohafkan, 2008) y en este sentido, la EAP da cuenta tanto de la agrobiodiversidad funcional (no intencional) como de la agrobiodiversidad planificada, ofreciendo posibilidades de planificación a través de la EAP potencial, con repercusiones a nivel de finca (producción) y también a nivel de matrices de agroecosistemas en unidades de paisaje a escalas más pequeñas (veredas, cuencas, municipios, regiones) (León-Sicard, 2010a).

\section{Parámetros para evaluar la EAP de los agroecosistemas mayores}

Los criterios propuestos por León-Sicard et al. (2012), Córdoba y León-Sicard (2013) y León-Sicard et al. (2014), tendientes a evaluar la EAP, corresponde a la sumatoria de los parámetros indicados en la Tabla 1.

Cada parámetro, se valora en una escala numérica del 1-10, cuya interpretación cualitativa corresponde a la siguiente ecuación (Ec. 1):

$$
\mathrm{EAP}=\begin{gathered}
\mathrm{CEEP}+\mathrm{ECE}+\mathrm{DCE}+\mathrm{ECI} \\
+\mathrm{DCI}+\mathrm{US}+\mathrm{MA}+\mathrm{OP}+\mathrm{PC}+\mathrm{CA}
\end{gathered}
$$

La escala de interpretación del estado de la EAP, se indica en la tabla 2.

\section{Tabla 2. Escalas del estado de la Estructura Agroecológica Principal.}

\begin{tabular}{|c|l|}
\hline Valor numérico & $\begin{array}{c}\text { Interpretación de la Estructura Agroecológica } \\
\text { Principal de la finca }\end{array}$ \\
\hline $80-100$ & Fuertemente desarrollada \\
\hline $60-80$ & Moderadamente desarrollada \\
\hline $40-60$ & Ligeramente desarrollada \\
\hline $20-40$ & $\begin{array}{l}\text { Débilmente desarrollada, con potencial cultural para } \\
\text { completarla }\end{array}$ \\
\hline$\leq 20$ & $\begin{array}{l}\text { Sin estructura o con estructura débilmente desarrollada, } \\
\text { sin potencial cultural para establecerla }\end{array}$ \\
\hline
\end{tabular}

Fuente: León-Sicard, 2014.

\section{La EAP como una estructura disipativa}

En los agroecosistemas, en tanto se consideran como sistemas alejados del equilibrio, se producen constantemente intercambios de materia y, flujos de energía e información con el entorno, siendo imposible obtener equilibrios finales y permanentes, debido a la continua entropía generada en sus procesos de transformación. Sin embargo, estos sistemas evolucionan adaptándose a su entorno, hasta que alcanzan la disipación más débil posible mediante mecanismos de auto-organización o autopoyesis (Maturana y Varela, 2004; Prigogine, 1983; Wagensberg, 1998).

Cuando estos sistemas enfrentan disturbios o fluctuaciones, por ejemplo, cuando los agroecosistemas se encuentran sometidos a cambios de temperatura,

Tabla 1. Parámetros evaluativos para estimar la Estructura Agroecológica Principal.

\begin{tabular}{|c|c|c|}
\hline Parámetro & Sigla & Descripción \\
\hline $\begin{array}{l}\text { Conexión con la estructura ecológica } \\
\text { principal del paisaje }\end{array}$ & CEEP & $\begin{array}{l}\text { Valora la distancia de la finca en relación con los fragmentos cercanos de vegetación natural, } \\
\text { principalmente coberturas boscosas y cuerpos de agua. }\end{array}$ \\
\hline Extensión de conectores externos & ECE & $\begin{array}{l}\text { Evalúa el porcentaje de la extensión lineal de las cercas vivas, presentes en el perímetro de las } \\
\text { fincas. }\end{array}$ \\
\hline Diversificación de conectores externos & DCE & Evalúa diversidad de las cercas vivas o setos localizados en el perímetro del agroecosistema mayor. \\
\hline Extensión de conectores internos & $\mathrm{ECl}$ & Evalúa el porcentaje de la extensión lineal de las hileras de vegetación, pero a nivel interno. \\
\hline Diversificación de conectores internos & $\mathrm{DCl}$ & Evalúa la diversificación de las cercas vivas internas. \\
\hline Usos y conservación del suelo & US & Este parámetro evalúa la distribución porcentual de diferentes coberturas. \\
\hline Manejo de arvenses & MA & Valora las prácticas y sistemas de manejo de las plantas arvenses. \\
\hline Otras prácticas de manejo & $\mathrm{OP}$ & $\begin{array}{l}\text { Es un indicador que expresa los tipos de sistemas productivos (ecológicos, convencionales, o en } \\
\text { transición) de cada finca. }\end{array}$ \\
\hline Percepción-Conciencia & $\mathrm{PC}$ & $\begin{array}{l}\text { Evalúa el grado de claridad conceptual y conciencia de los productores respecto a la } \\
\text { agrobiodiversidad. }\end{array}$ \\
\hline Nivel de capacidad para la acción & CA & $\begin{array}{l}\text { Evalúa las capacidades y posibilidades de los agricultores para establecer, mantener o mejorar su } \\
\text { EAP. }\end{array}$ \\
\hline
\end{tabular}

Fuente: León-Sicard, 2014. 
pluviosidad o ataques de insectos o patógenos y cuando estos disturbios en lugar de desaparecer aumentan, pueden experimentar una transformación estructural organizada, que les permitiría mantener su funcionalidad en el tiempo y el espacio, hacia un nuevo estado pasajero llamado "estructura disipativa". Esta estructura es la fluctuación amplificada, estabilizada por las interacciones con el ambiente, manteniéndose debido a que se nutre continuamente del flujo energético procedente del disturbio (Prigogine, 1983; Prigogine y Lefever, 1973). La formación y mantenimiento de la estructura disipativa requiere al menos tres condiciones: (1) el sistema debe ser abierto y puede intercambiar continuamente materia y energía con el ambiente externo; (2) El sistema debe estar en estado de no equilibrio o lejos del equilibrio, porque el no equilibrio es la fuente del orden; (3) Las interacciones no lineales así como ciertas ecuaciones dinámicas no lineales deben existir en el sistema (Deng et al., 2017).

Desde su promulgación, la teoría de las estructuras disipativas ha demostrado un gran alcance, logrando actualmente dar soporte a varios marcos teóricos en ingeniería, medicina, psicología, agricultura y ciencias humanas (Ahn, 1998; Almendro y Weber, 2012; Deng et al., 2017; Marchettini et al., 2010; Pulselli et al., 2009; Shvartsev, 2009; Zhao et al., 2000), convirtiéndose en una importante referencia del sistema científico moderno (Zhan y Sheng, 1998).

El concepto de estructura disipativa fue aplicado en la ecología, desde los años setenta del pasado siglo, para explicar las relaciones que se presentan entre los depredadores y sus presas (Segel y Jackson, 1972) y en el estudio de la estructura y las funciones biológicas a nivel genético (Martinez y Carlsson, 1974). En la década de 1980 se empleó para estudiar la evolución de los ecosistemas (Bazykin et al., 1983) y explicar las relaciones entre las especies y los ecosistemas (Emig, 1985). Mientras que para finales del siglo XX fue aplicado al análisis de la distribución de las plantas (Tuzinkevich y Frisman, 1990) y en el diseño de procesos para manejar y controlar plantas arvenses (Wang, 1992), por citar solo algunos ejemplos.

En resumen el enfoque teórico de las estructuras disipativas, se puede aplicar para explicar el comportamiento de sistemas biológicos, físicos, químicos y sociales y para estudiar la evolución de sus estructuras en términos de una cierta "adquisición de orden" (Wagensberg, 1998) y para el análisis de la resiliencia de sistemas.
Si el agroecosistema es considerado un sistema abierto que interactúa constantemente con el ambiente (físico, biótico, social, económico y cultural) intercambiando materia, energía e información, la EAP puede ser considerada también como una estructura disipativa, que permite al sistema aumentar o mejorar las posibilidades de mantener su funcionalidad, incorporando, disipando o utilizando la materia, energía o información proveniente del disturbio. En este sentido, por ejemplo, la mayor agrobiodiversidad disponible en una finca, permite a las distintas plantas ubicadas en estratos diversificados y con fenologías y genotipos diversos, tener mayor resistencia física a disturbios climáticos, pero también amplios abanicos de respuesta en términos económicos y sociales a disturbios de distintos orígenes y clase.

Dimensionar la EAP como una estructura disipativa, permite analizar los componentes del agroecosistema y fortalecer aquellos que pueden disipar el disturbio, de manera natural cuando el sistema ha madurado o intervenir sobre ellos de manera cultural. Es decir, ante un disturbio relacionado con el aumento de la evapotranspiración o la disminución de agua disponible, se pueden utilizar especies de mayor resistencia, utilización de coberturas verdes para proteger el suelo u otras estrategias, que de suyo son consecuencia de procesos sociales o económicos. Igualmente permitiría dirigir los esfuerzos a proponer soluciones de tipo cultural como el ahorro del agua, la captación de lluvias u otras, mediadas por las características culturales de las sociedades agrarias particulares.

\section{APORTES DE LA EAP AL ANÁLISIS DE LOS AGROECOSISTEMAS}

Asumiendo este enfoque teórico de la termodinámica, la EAP podría considerarse como una estructura disipativa de tipo cultural que, en la medida en que se incrementa, mejora las posibilidades de interacción y adaptación a diferentes tipos de disturbios, debido a la complejidad inherente a una mayor agrobiodiversidad y conectividad entre sectores de los agroecosistemas. De esta manera, la mayor EAP (estructurada) ayudaría a disipar los efectos negativos de factores externos sean estos culturales o ecosistémicos.

La EAP, fue propuesta en Colombia en el estudio de León-Sicard et al. (2011) quienes compararon seis fincas hortícolas ecológicas (de 20 a 0,16 hectáreas), en la sabana de Bogotá utilizando los diez criterios 
generales expuestos por León-Sicard (2010a). Se encontró valores de EAP entre 47 (ligeramente desarrollada) y 81 (fuertemente desarrollada), que revelaron diferencias sustanciales de manejo entre las fincas estudiadas y un alto grado de aislamiento de ellas con la estructura ecológica del paisaje. Este primer ejercicio de valoración de la EAP indicó que el índice podría ser aplicado en distintas condiciones socioeconómicas y en distintas regiones naturales del país. Para ello se realizaron nuevos estudios otras zonas de producción campesina de Colombia (Anolaima, Quipile y Pulí en el departamento de Cundinamarca), en agroecosistemas cafeteros de mayor biodiversidad.

En este sentido, Córdoba y León-Sicard (2013) estudiaron la resiliencia ecosistémica y cultural de seis agroecosistemas cafeteros (tres ecológicos y tres convencionales) en Anolaima (Andes colombianos), frente a la variabilidad climática. Todas las fincas ecológicas presentaron mejores condiciones de EAP y mayor resiliencia que las convencionales, aunque todas estaban ubicadas en una región caracterizada por la susceptibilidad a movimientos en masa y respondían a una historia común de poblamiento y de condiciones socioeconómicas similares. En otro estudio realizado en agroecosistemas cafeteros de Quipile y Pulí (Cundinamarca), Cepeda et al. (2014) valoraron la EAP en una zona con diferentes grados de intensificación agrícola, comparando los resultados con la metodología de tipificación de Moguel y Toledo (1999). En distintos tipos de coberturas los autores midieron variables locales asociadas al manejo agrícola (riqueza, densidad y altura promedio de árboles, densidad y altura promedio del café, riqueza de arvenses, porcentaje de cobertura del dosel y distancia al bosque más cercano como factor del paisaje) y determinaron los tipos y frecuencia de aplicación de agroquímicos y otras variables culturales de manejo.

Los autores encontraron una buena correlación de la clasificación de Moguel y Toledo (1999) con la clasificación propuesta en el estudio. Además, determinaron que la diversidad floral y de los espacios naturales cercanos al cultivo inciden sustancialmente en la riqueza de las abejas visitantes del café y con ello propusieron que la EAP, a través de arreglos productivos diversificados con alta conectividad interna y externa, propicia efectos sinérgicos que aumentan la riqueza de abejas dentro del cafetal y potencian su conservación y función ecológica de polinización.

Otro estudio realizado para explicar las razones socioambientales de los migrantes urbanos voluntarios hacia comunidades rurales intencionales (Pinzón, 2014), valoró los efectos ecosistémicos de esta migración a nivel de los cambios ocurridos en la EAP de las fincas, en tres ecoaldeas del departamento de Cundinamarca (Varsana ubicada en el municipio de Granada, El Retoño en el municipio de Silvania y Aldea Feliz en el municipio de San Francisco), utilizando técnicas etnográficas y muestreos de vegetación.

Los resultados mostraron que, los migrantes aportaron positivamente en el mejoramiento de la agrobiodiversidad de las fincas, medida a través de la EAP. Varsana (cuyos habitantes llegaron en 1979) pasó de una EAP inicial de 18 (débilmente desarrollada) a valores de 80 (fuertemente desarrollada) en 2013, El Retoño pasó de 26 a 81 en 15 años (1998-2013) y Aldea Feliz de 63 a 92 en 7 años (2006-2013). Tales modificaciones estuvieron ligadas a las motivaciones personales de los migrantes que buscan en ambientes diferentes al urbano, elementos de vida sana, satisfacción de aspiraciones espirituales y reivindicación de valores de solidaridad y respeto, acompañados de estructuras administrativas y económicas de tipo solidario.

Igualmente, y a fin de valorar el rol de la EAP en la resiliencia general de agroecosistemas citrícolas a la variabilidad climática en condiciones de la Orinoquia colombiana, Cleves-Leguizamo y Jarma-Orozco, 2014 y Cleves-Leguizamo et al. (2016) estudió esta característica en 18 fincas ubicadas en el piedemonte orinocense, encontrando alta correlación entre la EAP y la disminución en el número de controles fitosanitarios, realizados por los cultivadores de naranja (Citrus sinensis (L.) Osbeck) var. Valencia, al igual que correlaciones positivas con la productividad de las fincas estudiadas.

Finalmente, León-Sicard et al. (2014) realizaron un ejercicio preliminar para incluir agroecosistemas (más allá de la clasificación Corine Land Cover) en el mapa nacional de ecosistemas y para ello propusieron valorar la EAP como un criterio de conectividad agroecosistémica que puede ser representado inclusive en mapas a escala 1:100.000

Los ejemplos anteriores muestran que la Estructura Agroecológica Principal de los agroecosistemas mayores puede convertirse en una valiosa herramienta para el estudio y planificación del uso de los agroecosistemas, tanto a nivel de su manejo interno, como en asuntos relacionados con el ordenamiento y la planificación territorial. 
A nivel de predios o de escala de manejo en finca, la EAP facilita la comprensión de las interrelaciones que proporciona la agrobiodiversidad. Aunque aún no se han realizado estudios específicos en este campo; es posible adelantar la hipótesis de relaciones altas y positivas entre la EAP y la abundancia de polinizadores o enemigos naturales y de variadas relaciones intra $e$ interespecíficas de distintos organismos (responsables de ser agentes o vectores de enfermedades y daños en los cultivos). En esta categoría entra la biodiversidad edáfica, que puede ser mejorada al influjo del manejo de la agrobiodiversidad, vía refuerzo de la EAP.

Por otro lado, y como ya lo advirtiera León-Sicard (2014), la EAP puede ayudar en la planificación del uso de la finca, incorporando la noción de EAP potencial, es decir de la ubicación, trazado e implementación de mejores y más diversificados corredores internos y externos de vegetación acompañante, inclusive del manejo de parches de vegetación natural al interior de las fincas (ecosistemas al interior de agroecosistemas). La EAP permite calcular la selección de bancos de flores y la introducción de determinadas especies arbustivas o forestales, que ya han sido probadas como benéficas para la producción o el control de poblaciones de insectos, al estilo de los sistemas silvopastoriles intensivos (Nicholls et al., 2001; Van der Putten et al., 2001)

Por otra parte, la EAP brinda explicaciones, a escala de finca, de la conectividad del paisaje. Ya no se trata de considerar los corredores biológicos o los parches de vegetación o los bosques remanentes como piezas sueltas en el paisaje, sino que, la EAP permite agruparlos en torno a las unidades mismas de producción agropecuarias y por lo tanto visibiliza una serie de relaciones culturales (económicas, sociales, simbólicas y tecnológicas) ocultas desde el análisis parcial de la ecología del paisaje. En otras palabras, la EAP es la entrada de los productores agropecuarios en los mapas de la planificación ambiental del territorio.

La resiliencia socioecológica, reconoce que la cultura tiene un componente tecnológico que es incorporado por los agricultores en respuesta a los disturbios. Desde esta perspectiva, la caracterización de la EAP permite determinar qué prácticas culturales son necesarias para aumentar la agrobiodiversidad, por ejemplo, mediante el aumento en el número de especies vegetales y animales, el desarrollo de agroforestería, la práctica de alelopatía, la cosecha de agua, el manejo de coberturas y el uso de entomopatógenos; de manera que no sólo es posible aumentar los rendimientos, sino también la cantidad y variedad de alimentos, creándose fuertes conexiones con procesos de seguridad, soberanía y autonomía alimentaria, mejorando los procesos nutricionales de los agricultores y sus comunidades (Pirachican, 2014).

Los anteriores puntos demuestran la pertinencia de la evaluación de la EAP como una metodología de alta utilidad para la toma de decisiones, ya que sus resultados permiten a los agricultores efectuar ajustes en sus agroecosistemas, estimulando los flujos energéticos, de tal manera que al aumentar la conectividad y biodiversidad, se puede incrementar la resiliencia y productividad de sus unidades agrícolas y pecuarias y como consecuencia de esto sus ingresos; nivel de vida y autonomía alimentaria.

Por último, es importante señalar que los agricultores conocen muy bien todas las características particulares de sus fincas y de su entorno ecosistémico y por lo tanto, son los llamados a convertirse en agentes de cambio, aumentando no solo la resiliencia natural propia de cada agroecosistema, sino también la resiliencia adquirida, como resultado de su intervención en el ajuste de los parámetros constitutivos de la E.A.P.

A nivel administrativo la EAP se puede convertir en un insumo importante para el diseño de políticas públicas de desarrollo con enfoque territorial, partiendo de las necesidades locales con fundamento en una activa participación de los agricultores y, finalmente, también se puede convertir en un instrumento teórico-práctico de utilidad para avanzar en la taxonomía de los agroecosistemas, tema que por ahora no ha sido abordado por la agroecología.

Conflicto de intereses: el manuscrito fue preparado y revisado con la participación de los autores, quienes declaran no tener algún conflicto de interés que coloquen en riesgo la validez de los resultados aquí presentados.

\section{REFERENCIAS BIBLIOGRÁFICAS}

Ahn, H. 1998. Speculation in the financial system as a'dissipative structure. Seoul J. Econ. 11(3), 295-319.

Almendro, M. y D. Weber. 2012. Dissipative processes in psychology: From the psyche to totality. Int. J. Transpers Stud. 31(2), 1-22.

Altieri, A. 2009. El estado del arte de la agroecología: revisando avances y desafíos. pp. 77-102. En: León-Sicard, T. y M. Altieri (eds.). Vertientes del pensamiento agroecológico. Fundamentos y aplicaciones. Sociedad 
Científica Latinoamericana de Agroecología, Bogotá, Colombia.

Altieri, M.A.H., S. Liebman, M. Magdoff, F. Norgaard, R. Sikor y O. Thomas. 1999. Agroecología: Bases científicas para una agricultura sustentable. Nordan-Comunidad, Montevideo, Uruguay.

Altieri, M. y P. Koohafkan. 2008. Enduring farms: climate change, smallholders and traditional farming communities. Environment and development. Vol. 6. Third World Network (TWN), Kuala Lumpur, Malaysia.

Altieri, M. y C. Nicholls. 2009. Cambio climático y agricultura campesina: impactos y respuestas adaptativas. LEISA Revista Agroecol. 24(4), 5-8.

Ángel, A. 1993. La trama de la vida. Bases ecológicas del pensamiento ambiental. Ministerio de Educación Nacional, Instituto de Estudios Ambientales (IDEA), Universidad Nacional de Colombia, Bogotá, Colombia.

Ángel, A. 1995. La fragilidad ambiental de la cultura. Instituto de Estudios Ambientales, Universidad Nacional de Colombia, Bogotá, Colombia.

Ángel, A. 1996. El reto de la vida. Ecosistema y cultura. Una introducción al estudio del medio ambiente. Ecofondo, Bogotá, Colombia.

Bazykin, A., A. Khibnik y E. Aponin. 1983. A model of evolutionary appearance of dissipative structure in ecosystems. J. Math. Biol. 18(1), 13-23. Doi: 10.1007/ BF00275907

Cepeda, J., D. Gómez y C. Nicholls. 2014. La estructura importa: Abejas visitantes del café y Estructura Agroecológica Principal (EAP) en cafetales. Rev. Colomb. Entomol. 40(2), 241-250.

Cleves-Leguizamo, J.A. y A. Jarma-Orozco. 2014. Characterization and typification of citrus production systems in the department of Meta. Agron. Colomb. 32(1), 113-121. Doi: 10.15446/agron.colomb.v32n1.42164

Cleves-Leguizamo, A., J. Toro y T. León-Sicard. 2016. La estructura agroecológica principal (EAP). Metodología para analizar la biodiversidad y resiliencia en agroecosistemas. pp. 1-26. En: Memorias Congreso Nacional de Medio Ambiente (CONAMA). 2016. Fundación Conama, Madrid, España.

Córdoba, C. y T. León-Sicard. 2013. Resiliencia de sistemas agrícolas ecológicos y convencionales frente a la variabilidad climática en Anolaima (Cundinamarca-Colombia). Agroecol. 8(1), 21-32.

Dalgaard, T., T. Nicholas, J. Hutchings, y R. Porter. 2003. Agroecology, scaling and interdisciplinary. Agric. Ecosyst. Environ. 100(1), 39-51. Doi: 10.1016/ S0167-8809(03)00152-X

Deng, X., S. Zheng, P. Xu y X. Zhang. 2017. Study on dissipative structure of China's building energy service industry system based on brusselator model. J. Clean. Prod. 150, 112-122. Doi: 10.1016/j.jclepro.2017.02.198

Duarte, O. 1990. Tipificación de fincas en la comarca de San Gil, Colombia, con base en una encuesta dinámica. pp. 201-220. En: Tipificación de sistemas de producción agrícola. Escobar \& Berdegué Editores, Santiago de Chile, Chile.

Emig, C. 1985. Relations entre l'espèce, structure dissipatrice biologique, et l'écosystème, structure dissipatrice écologique. Contribution à la théorie de l'évolution des systèmes non-en équilibre. C. R. Acad. Sci. Paris 300, 3(8), 323-326.

Escobar, G. y J. Berdegué. 1990. Conceptos y Metodología para la tipificación de sistemas de finca: la experiencia de RIMISP. pp. 13-44. En: Escobar, G. y J. Berdegué (eds.). Tipificación de sistemas de producción agrícola. Centro Latinoamericano para el Desarrollo Rural, Santiago de Chile, Chile.

Fonseca, J. y J.A. Cleves-Leguizamo. 2015. Agroecología y cambio climático: dos retos urgentes para la humanidad. pp. 69-81. En: Cabrera O., M.I (ed.). Ciudadanía ambiental, crisis de la agricultura convencional y desafíos para una agroecología orientada hacia el desarrollo rural. Universidad Nacional Abierta y a Distancia (UNAD), Bogotá, Colombia.

Gliessman, S. 1990. Agroecology: researching the ecological basis for sustainable agricultura. pp. 3-10. En: Gliessman, S. (ed.). Agroecology. Ecological studies. Vol 78. New York, NY. Doi: 10.1007/978-1-4612-3252-0_1

León-Sicard, T. 2014. Perspectiva ambiental de la agroecología: la ciencia de los agroecosistemas. Serie IDEAS 23. Instituto de Estudios Ambientales, Universidad Nacional de Colombia, Bogotá, Colombia.

León-Sicard, T. 2007. Medio ambiente y tecnología y modelos de agricultura en Colombia. Hombre y arcilla. ECOE-IDEA, Universidad Nacional de Colombia, Bogotá, Colombia.

León-Sicard, T. 2010a. Agroecología: desafíos de una ciencia ambiental en construcción. pp. 53-77. En: León-Sicard, T. y M. Altieri (eds.). Vertientes del pensamiento agroecológico: fundamentos y aplicaciones. Sociedad Científica Latinoamericana de Agroecología; Universidad Nacional de Colombia, Bogotá, Colombia.

León-Sicard, T. 2010b. Regulación biológica en agricultura de pequeña escala: un enfoque desde la sostenibilidad. pp. 271-293. En: León-Sicard, T. y M. Altieri (eds.). Vertientes del pensamiento agroecológico: fundamentos y aplicaciones. Sociedad Científica Latinoamericana de Agroecología; Universidad Nacional de Colombia, Bogotá, Colombia.

León-Sicard, T. N. Clavijo, C. Córdoba y L. Gomajoa. 2014. Lineamientos conceptuales y metodológicos para cartografiar agroecosistemas en el mapa nacional de 
ecosistemas de Colombia (escala 1:100.000). IDEAM, Instituto de Estudios Ambientales (IDEA), Universidad Nacional de Colombia, Bogotá, Colombia.

León-Sicard, T., T. Mendoza y C. Córdoba. 2014. La Estructura Agroecológica Principal de la finca (EAP): un concepto útil en agroecología. Agroecol. 9(1-2), 55-66.

Marchettini, N., E. Del Giudice, V. Voeikov y E. Tiezzi. 2010. Water: A medium where dissipative structures are produced by a coherent dynamics. J. Theor. Biol. 265(4), 511-516. Doi: 10.1016/j.jtbi.2010.05.021

Martinez, H. y G. Carlsson. 1974. Genetic nets and dissipative structures: An algebraic approach. Bull. Math. Biol. 36(2), 183-196. Doi: 10.1007/BF02458602

Martínez, R. 2002. Agroecología: atributos de sustentabilidad. InterSedes: Revista de las Sedes Regionales 3(5), 25-45.

Maturana, H. y F. Varela. 2004. De máquinas y seres vivos: autopoiesis: la organización de lo vivo. pp. 73-74. Editorial Universitaria Lumen, Buenos Aires, Argentina.

Méndez, E. y S. Gliessman. 2002. Un enfoque interdisciplinario para la investigación en agroecología y desarrollo rural en el trópico latinoamericano. Manejo Integrado de Plagas y Agroecología 64, 5-16.

Moguel, P. yV. Toledo. 1999. Biodiversity conservation in traditional coffee systems in Mexico. Rev. Conserv. Biol. 13(1), 11-21. Doi: 10.1046/j.1523-1739.1999.97153.x

Nicholls, C.I., M. Parrilla, y M.A. Altieri. 2001. The effects of a vegetational corridor on the abundance and dispersal of insect biodiversity within a northern California organic vineyard. Landsc. Ecol. 16(2), 133-146. Doi: 10.1023/A:1011128222867

Pinzón, M. 2014. Transformación de la Estructura Agroecológica Principal en comunidades intencionales rurales (Eco aldeas). Tesis de maestría. Facultad de Ciencias Económicas, Universidad Nacional de Colombia, Bogotá, Colombia.

Pirachican, E. 2014. Autonomía alimentaria en sistemas agrícolas ecológicos y convencionales en Anolaima (Cundinamarca). Tesis de maestría. Facultad de Ciencias Económicas, Universidad Nacional de Colombia, Bogotá, Colombia.

Prigogine, I. 1983. ว̇Tan Solo Una Ilusion? Tusquets Editores S.A., Barcelona, España.

Prigogine, I. y R. Lefever. 1973. Theory of Dissipative Structures. pp. 124-135. En: Haken, H. (ed.). Synergetics: Cooperative Phenomena in multi-component systems. Vieweg Teubner Verlag, Wiesbaden, Alemania. Doi: 10.1007/978-3-663-01511-6_10

Pulselli, R.M., E. Simoncini y E. Tiezzi. 2009. Self-organization in dissipative structures: A thermodynamic theory for the emergence of prebiotic cells and their epigenetic evolution. Biosyst. 96(3), 237-241. Doi: 10.1016/j.biosystems.2009.02.004

Segel, L.A. y J.L. Jackson. 1972. Dissipative structure: An explanation and an ecological example. J. Theor. Biol. 37, 545-559. Doi: 10.1016/0022-5193(72)90090-2

Shvartsev, S.L. 2009. Self-organizing abiogenic dissipative structures in the geologic history of the earth. Earth Sci. Front. 16(6), 257-275. Doi: 10.1016/ S1872-5791(08)60114-1

Toledo, V. 1990. Modernidad y ecología: La nueva crisis planetaria. Ecol. Polít. 3, 9-22.

Tuzinkevich, A.V y Y.Y Frisman. 1990. Dissipative structures and patchiness in spatial distribution of plants. Ecol. Model. 52(3-4), 207-224. Doi: 10.1016/0304-3800(90)90016-A

Van der Hammen, T. 1998. Plan ambiental de la Cuenca Alta del río Bogotá (Análisis y orientaciones para el ordenamiento territorial). Corporación Autónoma Regional de Cundinamarca, CAR, Bogotá, Colombia.

Van der Hammen, T. y G. Andrade. 2003. Estructura ecológica principal de Colombia, primera aproximación. Ministerio de Ambiente, Vivienda y Desarrollo Territorial, Instituto de Hidrología, Meteorología y Estudios Ambientales, Bogotá, Colombia.

Van der Putten, W.H., L. Vet., J. Harvey y F. Wackers. 2001. Linking above- and belowground multitrophic interactions of plants, herbivores, pathogens, and their antagonists. Trends Ecol. Evol. 16(10), 547-554. Doi: 10.1016/S0169-5347(01)02265-0

Wagensberg, J. 1998. Ideas sobre la complejidad del mundo. $4^{\mathrm{a}}$ ed. Tusquets Editores S.A., Barcelona, España.

Wang, J. 1992. Dissipative structure theory and strategy for systematic control of field weeds. Chinese J. Ecol. 11(3), 49-52.

Zhan, K. y X. Sheng. 1998. Prigogine and theory of dissipative structure ( $2^{\text {nd }}$ ed.). Shaanxi University of Science and Technology, Technique Publishing House, Shaanxi, China.

Zhao, C., B.E. Hobbs, H.B Mühlhaus y A. Ord. 2000. Finite element modelling of dissipative structures for nonequilibrium chemical reactions in fluid-saturated porous media. Comput. Meth. Appl. Mech. Eng. 184 (1), 1-14. Doi: 10.1016/S0045-7825(99)00449-1 